\title{
Ictiofauna da microbacia do Rio Jaguari, Juaguari/RS, Brasil
}

\author{
Carlos Eduardo Copatti ${ }^{1,2}$, Lucéle Gonçalves Zanini ${ }^{1}$ \& André Valente ${ }^{1}$ \\ ${ }^{1}$ Departamento de Ciências Biológicas, Universidade de Cruz Alta - UNICRUZ, \\ Rua Andrade Neves, 308, CEP 98025-810, Cruz Alta, RS, Brasil, \\ e-mail: lucele.zanini@gmail.com, andrepelucio@yahoo.com.br \\ ${ }^{2}$ Autor para correspondência: Carlos Eduardo Copatti, e-mail: carloseduardocopatti@yahoo.com.br
}

COPATTI, C.E., ZANINI, L.G. \& VALENTE. A. Ichthyofauna of the Jaguari river microbasin, Jaguari /RS, Brazil. Biota Neotrop., 9(2): http://www.biotaneotropica.org.br/v9n2/en/abstract?inventory+bn00809022009.

\begin{abstract}
The microbasin of the Jaguari river belongs to the hydrographic basin of the River Uruguay and has its estuary in the raised edge of the river Ibicuí. This work had the objectif identify the existing ictyofaun of the microbasin of the river Jaguari in Jaguari-RS. For the accomplishment of the research identifications of units captured in the four months of the year had been carried through, being that nets of wait of each one of following meshes (measured in $\mathrm{cm}$ between adjacent points): $1.0 ; 1.5 ; 2.0 ; 2.5 ; 3.0 ; 4.0 ; 5.0 ; 6.0$; and 7.0. The wait nets had remained in the water for 24 hours, being revised to each six hours, always in same periods (06:00 AM; 12:00 AM; 06:00 PM and 12:00 PM hours). The collected fish, after to be identified, had been numbered and then after, fixed in formaldehyde conserved $4.0 \%$ and later in alcohol 70.0\%. A total of 572 individuals in the four stations of the year were identified, distributed in 14 families and 26 species. In the spring the biggest capture occurred, with 156 individuals, distributed in 15 species; in the period of summer the biggest richness occurred, with a capture of 146 individuals distributed in 19 species; in the autumn and winter a capture of 135 individuals for both occurred the stations, with a richness of 16 and 12 species, respectively. The species most abundant, with 291 units, was Apareiodon affinis, being that Hemiancistrus fuliginosos (75), Hypostomus commersoni (50) and Steindachnerina brevipina (41) also had high representation.
\end{abstract}

Keywords: diversity, Uruguay river, fishes.

COPATTI, C.E., ZANINI, L.G. \& VALENTE. A. Ictiofauna da microbacia do rio Jaguari, Jaguari/RS, Brasil. Biota Neotrop., 9(2): http://www.biotaneotropica.org.br/v9n2/pt/abstract?inventory+bn00809022009.

Resumo: A microbacia do rio Jaguari pertence à Bacia Hidrográfica do rio Uruguai e tem sua foz na margem superior do rio Ibicuí. Este trabalho teve o objetivo de identificar a Ictiofauna existente em um trecho da microbacia do rio Jaguari, em Jaguari-RS. Para a realização da pesquisa foram realizadas identificações de exemplares capturados nas quatro estações do ano, através do uso de redes de espera das seguintes malhas (medidas em $\mathrm{cm}$ entre nós adjacentes): 1,$0 ; 1,5 ; 2,0 ; 2,5 ; 3,0 ; 4,0 ; 5,0 ; 6,0 ;$ e 7,0. As redes de espera permaneceram na água por 24 horas, sendo revisadas a cada seis horas, sempre nos mesmos horários (06:00; 12:00; 18:00 e 24:00 horas). Os peixes coletados, após serem identificados, foram numerados e, logo após, fixados em formol a 4,0\% e depois conservados em álcool 70,0\%. Foram identificados um total de 572 indivíduos, distribuídos em 14 famílias e 26 espécies. Na primavera ocorreu a maior captura, com 156 indivíduos, distribuídos em 15 espécies; no verão ocorreu a maior riqueza, com 146 indivíduos distribuídos em 19 espécies; já no outono e inverno ocorreu uma captura de 135 indivíduos para ambas as estações, com 16 e 12 espécies, respectivamente. A espécie mais abundante, com 291 exemplares, foi Apareiodon affinis, sendo que Hemiancistrus fuliginosos (75), Hypostomus commersoni (50) e Steindachnerina brevipina (41) também tiveram alta representatividade.

Palavras-chave: diversidade, rio Uruguai, peixes. 


\section{Introdução}

No Rio Grande do Sul distinguem-se, basicamente, dois grupos de cursos d'água, os que deságuam no Atlântico e os que deságuam no rio Uruguai. A região do Planalto Médio, hidrograficamente pertence à bacia do rio Uruguai (Vieira 1984). A microbacia do rio Jaguari pertence à Bacia Hidrográfica do rio Ibicui, a maior bacia da região hidrográfica Uruguai.

No sistema do rio Uruguai, trabalhos com a comunidade ictiofaunística são relativamente escassos (Weis et al. 1983, Bertoletti 1985, Bossemeyer et al. 1985, Bertoletti et al. 1989a,b, 1990, Hahn et al. 1997). Di Persia \& Neiff (1980) consideraram incipiente o estudo da ictiofauna deste sistema hidrográfico quando comparado aos rios Paraná e Paraguai.

O conhecimento da composição da ictiofauna e a compreensão dos mecanismos funcionais da mesma constituem condições imprescindíveis para a avaliação das possíveis alterações ambientais e a definição de medidas mitigadoras dos impactos sobre o ambiente e seus diversos componentes (Hickson et al. 1995). Buckup et al. (2007) registraram a ocorrência de 2.587 espécies de peixes de água doce distribuídos em 39 famílias no Brasil, o que demonstra a alta biodiversidade da região neotropical e afirma o aumento do conhecimento em relação à diversidade de peixes no Brasil.

Trabalhos de levantamento faunístico são o passo inicial indispensável para o estudo biológico e manejo de uma área, por fornecerem informações básicas para uma série de outros trabalhos científicos (Casatti et al. 2001). A determinação da biodiversidade das assembléias de peixes e dos seus padrões de variação espaciais e temporais é de grande relevância para avaliar a qualidade ambiental, uma vez que os peixes ocupam variadas posições na teia trófica e o monitoramento biológico da ictiofauna em rios também é essencial para identificar respostas do ambiente aos impactos causados pela ação antrópica (Amorin et al. 1983).

Neste contexto, objetivou-se identificar a Ictiofauna existente em um trecho da microbacia do rio Jaguari, Jaguari-RS. Conhecer a estrutura da comunidade e a diversidade das espécies irá gerar importantes dados ecológicos que servirão como subsídio para o manejo adequado dos recursos naturais, bem como para trabalhos futuros nas áreas de ictiologia, ecologia e piscicultura.

\section{Material e Métodos}

$\mathrm{O}$ rio Jaguari tem sua foz à margem direita do rio Ibicui e seus tributários são pequenos arroios e riachos de curso rápido, formando vales com afloramentos rochosos característicos. Os locais de coleta situam-se nos limites do município de Jaguari e São Francisco de Assis/RS. A ictiofauna dos dois trechos da microbacia foi amostrada entre a primavera de 2006 e o inverno de 2007, através de uma coleta em cada época do ano. Os trechos distanciavam-se em aproximadamente $500 \mathrm{~m}$ entre si apresentando características distintas. As margens de ambos os locais de coleta possuem mata ripária muito escassa e, em muitos casos, inexistente.

O primeiro trecho encontra-se no rio Rosário, na localidade conhecida como "Jaguarizinho" em Jaguari-RS, o qual tem sua nascente em Santiago-RS e sua foz no rio Jaguari, em Jaguari-RS, municípios localizados na região central do Estado do Rio Grande do Sul. O local escolhido situa-se nos limites do município de Jaguari e São Vicente do Sul-RS, com as seguintes coordenadas geográficas: $29^{\circ} 30^{\prime} 45,13^{\prime \prime}$ S e $54^{\circ} 46^{\prime} 15,48^{\prime \prime}$ W. Este trecho tem uma largura de aproximadamente $30 \mathrm{~m}$, com profundidade média de $1,5 \mathrm{~m}$, possui leito muito acidentado, com fortes correntezas. O fundo do leito possui pouco lodo e material em decomposição, uma vez que é composto por uma grande quantidade de cascalhos e lages de pedras.
O segundo trecho encontra-se na localidade denominada "Poço Fundo" em Jaguari-RS, possuindo uma largura de aproximadamente $50 \mathrm{~m}$ e profundidade média de $6 \mathrm{~m}$. As coordenadas geográficas são: $29^{\circ} 30^{\prime} 54,81^{\prime}$ ' S e $54^{\circ} 45^{\prime} 50,38^{\prime}$ 'W. Nessa área, o fundo apresenta-se lodoso e com acúmulo de sedimentos.

Foi realizada uma coleta em cada ponto de amostragem nos meses de outubro e dezembro de 2006 e abril e agosto de 2007. Foram utilizadas redes de espera de $20 \mathrm{~m}$ de comprimento e $1 \mathrm{~m}$ de altura, com as seguintes malhas (medidas em $\mathrm{cm}$ entre nós adjacentes): 1,0; 1,$5 ; 2,0 ; 2,5 ; 3,0 ; 4,0 ; 5,0 ; 6,0$ e 7,0, as quais foram instaladas com auxilio de um barco a remo de alumínio de 4,2 m. As redes foram colocadas em regiões diversas dos pontos de amostragem, de forma aleatória, abordando margem e leito.

As redes permaneceram na água por 24 horas, sendo revisadas a cada seis horas $(6: 00 ; 12: 00 ; 18: 00$ e 24:00). Os peixes coletados foram numerados com auxílio de rotulador de letras (Rotex 1760 office) e fitas vinílicas para gravação e, logo após, fixados em formol 10\%, onde permaneceram por 72 horas, sendo então transferidos para álcool $70 \%$ conforme Malabarba \& Reis (1987). Os exemplares encontramse acondicionados no Laboratório de Ecologia da Universidade de Cruz Alta (UNICRUZ, RS), tendo apenas caráter didático.

Para cada exemplar capturado foram registradas, no campo, as seguintes informações: data; estação de amostragem; aparelho de pesca e; período de captura.

Oxigênio dissolvido e temperatura foram medidos utilizandose oxímetro YSI (modelo Y5512), pH através de pHmetro Quimis (modelo 400.A) e dureza total da água segundo Adad (1982). Amônia total (método de Nessler) conforme Boyd (1982) e alcalinidade total segundo Adad (1982). Tais medidas foram realizadas no Laboratório de Fisiologia de Peixes da Universidade Federal de Santa Maria (UFSM), exceto oxigênio e temperatura que foram medidos no momento das coletas. As medidas de peso e comprimento foram realizadas no Laboratório de Aqüicultura da Universidade Regional Integrada (URI) - Campus Santiago. Para medidas de peso utilizouse uma balança eletrônica, modelo AS 2000, capacidade $2.000 \mathrm{~g}$ e sensibilidade de $0,01 \mathrm{~g}$.

A diversidade específica para os ambientes foi calculada através do Índice de diversidade de Shannon (H') e eqüitabilidade de Pielou (J'), bem como riqueza total (S) e dominância da espécie mais abundante (k) com auxílio do programa Bio Diversity-Pro (1997).

Para determinação das espécies residentes e espécies migrantes foi utilizada a constância de ocorrência (Dajoz 1973), de fórmula: $\mathrm{C}=\mathrm{p} / \mathrm{P} \times 100$, onde $\mathrm{C}=$ valor de constância da espécie, $\mathrm{p}=$ número de coletas contendo a espécie estudada e $\mathrm{P}=$ número total de coletas efetuadas. Neste estudo, uma espécie foi considerada constante quando apresentou $\mathrm{C}>50 \%$, acessória, quando $25 \%<\mathrm{C} \leq 50 \%$ e acidental, quando $\mathrm{C} \leq 25 \%$.

\section{Resultados}

Nos dois locais amostrados foram coletados 572 indivíduos, distribuídos em 14 famílias e 26 espécies (Tabela 1). Em outubro ocorreu a maior captura, com 156 indivíduos, distribuídos em 15 espécies, não diferindo muito de dezembro, que apresentou 146 indivíduos e 19 espécies. Em abril e agosto foram coletados 135 indivíduos em ambos os meses, distribuídos em 16 e 13 espécies, respectivamente.

Dentre os horários de coleta, a maior abundância ocorreu entre as 18:00 e 24:00 horas. A maior abundância de capturas foi com as redes de malhas entre 1,0-2,0 cm entre nós adjacentes (Tabela 1).

A família Loricaridae foi a mais representada nesse estudo, com quatro espécies, seguida das famílias Characidae, Cichlidae e Pimelodidae, com três espécies cada. Para Loricaridae, citamse: Hypostomus commersoni (Valenciennes 1836) $(8,74 \%)$, 
Tabela 1. Espécies identificadas na Microbacia do rio Jaguari. Época do ano: outubro (O); dezembro (D); abril (Ab); agosto (Ag). Horário: 00:00-06:00 horas (1); 06:00-12:00 horas (2); 12:00-18:00 horas (3); 18:00-24:00 horas. Malha: 1,0-2,0 (a); 2,5-4,0(b); 5,0-7,0(c).

Table 1. Species identified in the Microbasin of the river Jaguari. Time of year: October (o); December (D); April (AP); August (Ag). Hour: 0:00-6:00 AM (1); 6:00-12:00 AM (2); 12:00-06:00 PM (3); 6:00-12:00 PM. Net: 1,0-2,0 (a); 2,5-4,0(b); 5,0-7,0(c).

\begin{tabular}{|c|c|c|c|c|c|c|c|c|c|c|}
\hline \multirow[t]{2}{*}{ Espécie } & \multicolumn{4}{|c|}{ Poço Fundo } & \multicolumn{4}{|c|}{ Jaguarizinho } & \multirow[t]{2}{*}{ Hora } & \multirow[t]{2}{*}{ Malha } \\
\hline & $\mathbf{O}$ & D & $\mathbf{A b}$ & Ag & $\mathbf{O}$ & D & $\mathbf{A b}$ & Ag & & \\
\hline \multicolumn{11}{|l|}{ CHARACIFORMES } \\
\hline \multicolumn{11}{|l|}{ PARADONTIDAE } \\
\hline Apareiodon affinis & 25 & 65 & 24 & 48 & 61 & 8 & 37 & 23 & $1,2,3,4$ & $a, b$ \\
\hline \multicolumn{11}{|l|}{ CURIMATIDAE } \\
\hline Steindachenerina brevipina & 15 & 7 & 0 & 2 & 8 & 2 & 7 & 0 & $1,2,3,4$ & $a, b$ \\
\hline Steindachenerina biornata & 1 & 0 & 0 & 0 & 0 & 0 & 0 & 0 & 1 & $\mathrm{~b}$ \\
\hline \multicolumn{11}{|l|}{ PROCHILODONTIDAE } \\
\hline Prochilodus lineatus & 0 & 0 & 0 & 0 & 0 & 0 & 2 & 1 & $1,2,3$ & $\mathrm{c}$ \\
\hline \multicolumn{11}{|l|}{ ANOSTOMIDAE } \\
\hline Schizodon nasutus & 0 & 1 & 0 & 0 & 0 & 1 & 0 & 0 & 1,4 & $a, b$ \\
\hline Leporinus striatus & 0 & 0 & 0 & 0 & 4 & 0 & 0 & 3 & 1,3 & $a, b$ \\
\hline \multicolumn{11}{|l|}{ CHARACIDAE } \\
\hline \multicolumn{11}{|l|}{ Characinae } \\
\hline Astyanax jacuhiensis & 2 & 7 & 2 & 1 & 1 & 3 & 2 & 2 & $1,2,3,4$ & $a, b$ \\
\hline Astyanax fasciatus & 0 & 0 & 0 & 0 & 0 & 1 & 0 & 0 & 4 & $\mathrm{a}$ \\
\hline Galeocharax humeralis & 0 & 1 & 0 & 0 & 0 & 0 & 0 & 0 & 1 & $\mathrm{~b}$ \\
\hline Serrasalminae & & & & & & & & & & a \\
\hline Serrasalmus maculatus & 1 & 0 & 0 & 0 & 0 & 1 & 0 & 0 & 1 & \\
\hline \multicolumn{11}{|l|}{ ACESTRORHYNCHIDEAE } \\
\hline Acestrorhynchus pantaneiro & 3 & 0 & 2 & 2 & 0 & 1 & 4 & 0 & $1,2,3,4$ & $\mathrm{a}, \mathrm{b}, \mathrm{c}$ \\
\hline \multicolumn{11}{|l|}{ ERYTHRINIDAE } \\
\hline Hoplias malabaricus & 0 & 2 & 0 & 0 & 1 & 1 & 1 & 0 & $1,3,4$ & $a, b$ \\
\hline Hoplias lacerdae & 0 & 0 & 0 & 0 & 0 & 0 & 2 & 0 & 2 & $\mathrm{~b}$ \\
\hline \multicolumn{11}{|l|}{ SILURIFORMES } \\
\hline \multicolumn{11}{|l|}{ LORICARIIDAE } \\
\hline Hypostomus commersoni & 6 & 8 & 1 & 13 & 6 & 3 & 0 & 13 & $1,2,3,4$ & \\
\hline Hemiancistrus fuliginosos & 4 & 5 & 12 & 11 & 8 & 2 & 26 & 7 & $1,2,3,4$ & \\
\hline Hypostomus roseopuntactus & 0 & 0 & 0 & 0 & 2 & 0 & 0 & 0 & 2 & \\
\hline Loricariichthys anus & 0 & 0 & 0 & 0 & 0 & 0 & 1 & 0 & 3 & \\
\hline \multicolumn{11}{|l|}{ HEPTAPTERIDAE } \\
\hline Rhamdia quelen & 1 & 0 & 0 & 1 & 0 & 0 & 2 & 1 & $1,2,3$ & $\mathrm{~b}, \mathrm{c}$ \\
\hline \multicolumn{11}{|l|}{ PIMELODIDAE } \\
\hline Pimelodus maculatus & 5 & 0 & 1 & 1 & 0 & 1 & 0 & 0 & 1,4 & $a, b, c$ \\
\hline Iheringichthys labrosus & 0 & 5 & 0 & 0 & 0 & 3 & 1 & 1 & $1,2,4$ & $a, b$ \\
\hline Pimelodus atrobrunneus & 0 & 0 & 3 & 0 & 0 & 2 & 2 & 0 & 1,3 & a \\
\hline \multicolumn{11}{|l|}{ AUCHENIPTERIDAE } \\
\hline Auchenipterus osteomystax & 0 & 3 & 0 & 0 & 0 & 0 & 0 & 0 & 2 & a \\
\hline \multicolumn{11}{|l|}{ PERCIFORMES } \\
\hline SCIAENIDAE & & & & & & & & & & \\
\hline Pachyurus bonariensis & 0 & 4 & 0 & 0 & 0 & 1 & 0 & 0 & $1,2,4$ & $a, b$ \\
\hline CICHLIDAE & & & & & & & & & & \\
\hline Gymnogeophagus gymnogenys & 0 & 2 & 0 & 0 & 1 & 0 & 1 & 2 & $1,2,3$ & $\mathrm{a}, \mathrm{b}, \mathrm{c}$ \\
\hline Crenicichla missioneira & 0 & 2 & 0 & 1 & 1 & 2 & 2 & 2 & $1,2,3,4$ & $a, b$ \\
\hline Crenicichla celidochilus & 0 & 1 & 0 & 0 & 0 & 1 & 0 & 0 & 2,3 & $\mathrm{a}$ \\
\hline Total & 63 & 113 & 45 & 80 & 93 & 33 & 90 & 55 & $(572)$ & \\
\hline
\end{tabular}


Hypostomus roseopuntactus (Reis, Weber \& Malabarba 1990) $(0,35 \%)$, Hemiancistrus fuliginosos (Cardoso \& Malabarba 1999) $(13,11 \%)$ e Loricariichthys anus (Valenciennes 1836) (0,17\%). Já para Cichlidae ocorrem Crenicichla missioneira (Lucena \& Kullander 1992) (1,75\%), Crenicichla celidochilus (Casciotta 1987) (0,35\%) e Gymnogeophagus gymnogenys (Hensel 1870) $(1,05 \%)$. Para Characidae: Characinae citam-se Astyanax fasciatus (Cuvier, 1819) (0,17\%), Astyanax jacuhiensis (Cope 1894) (3,5\%), Galeocharax humeralis (Valenciennes 1834) (0,17\%), enquanto que para Pimelodidae, Iheringichthys labrosus (Lütken, ex Kröyer 1874) (1,75\%), Pimelodus maculatus (LaCepède 1803) (1,4\%) e Pimelodus atrobrunneus (Vidal \& Lucena 1999) (1,22\%). Em conjunto as três famílias representaram $50 \%$ da riqueza total.

Três famílias apresentam uma riqueza de duas espécies cada: Curimatidae, com Steindachnerina biornata (Braga \& Azpelucueta 1987) $(0,17 \%)$ e Steindachnerina brevipina (Eigenmann \& Eigenmann 1889) (7,17\%); Erythrinidae com Hoplias lacerdae (Miranda Ribeiro 1908) (0,35\%) e Hoplias malabaricus (Bloch 1794) $(0,87 \%)$ e Anostomidae com Schizodon nasutus (Kner 1858) (0,35\%) e Leporinus striatus (Kner 1858) (1,22\%).

Sete famílias foram representadas apenas por uma espécie. Heptapteridae: Rhamdia quelen (Quoy \& Gaimard 1824) (0,87\%), Characidae: Serrasalminae: Serrasalmus maculatus (Kner 1858) (0,35\%), Paradontidae: Apareiodon affinis (Steindachner 1879) (50,87\%), Acestrorhynchideae: Acestrorhynchus pantaneiro (Menezes 1992) (2,1\%), Sciaenidae: Pachyurus bolnariensis (Steindachner 1879) (0,87\%), Auchenipteridae: Auchenipterus osteomystax (Miranda Ribeiro 1920) (0,53\%) e, Prochilodontidae: Prochilodus lineatus (Valenciennes 1836) (0,53\%).

A espécie mais abundante foi $A$. affinis, com 50,87\% dos exempares, seguida de $H$. fuliginosos com $13,11 \%, H$. commersoni com $8,74 \%$ e $S$. brevipina com $7,16 \%$.

As espécies $H$. fuliginosos e A. affinis foram capturadas nas quatro coletas em ambas as localidades. Entretanto as espécies $H$. roseopunctatus, $S$. biornata e $L$. striatus foram encontradas apenas em outubro, enquanto que A. fasciatus, G. humeralis, $C$. celidocheilus, $P$. bonariensis, S. nasutus e A.osteomystax o foram, apenas em dezembro. Já $L$. anus e H. lacerdae foram encontradas apenas em abril.

Espécimes de H. commersoni; H. fuliginosus; A. affinis; S. brevipina; A. jacuhiensis; A. pantaneiro e; C. missioneira foram capturadas em todos os horários amostrados, porém outras espécies como I. labrosus, $R$. quelen, G. gymnogenys, P. bonariensis, $H$. malabaricus e $P$. lineatus demonstraram atividade em horários distintos.

A maioria dos indivíduos foi coletada nas redes de menor malha (1,0-2,0 cm). Algumas espécies, como: R. quelen; S. maculatus; $S$. biornata; G. humeralis; $H$. lacerdae e; P. lineatus, foram apenas de redes de malhas superiores a $2,5 \mathrm{~cm}$, não havendo registros para redes de malhas inferiores a isso.

A diversidade H' apresentou-se superior para o mês de dezembro $(0,86)$ em comparação com os demais: outubro $(0,70)$, abril $(0,74)$ e agosto $(0,67)$ (Tabela 2$)$. Em dezembro a uniformidade dos organismos também foi maior, visto que a equitabilidade $\mathrm{J}$ ' nesta estação $(0,67)$ foi superior as demais,0,61 em abril e 0,60 em outubro e agosto (Tabela 2). Em todas coletas, a dominância $\mathrm{k}$ da espécie mais abundante no período foi superior a $45,18 \%$ (Tabela 2) o que influenciou uma redução da diversidade H' e da equitabilidade J'.

Os peixes com atividade entre 00:00 e 06:00 horas, apresentaram maior diversidade H' $(1,07)$, equitabilidade J' $(0,81)$, riqueza total (21) e menor dominância $\mathrm{k}(29,32 \%)$ em relação aos outros horários de coleta analisados (Tabela 3). A preferência pela atividade em horário noturno pela maioria das espécies é reforçada pelo fato da segunda maior riqueza (16) ter ocorrido para peixes ativos entre 18:00 e 0:00, registrando, igualmente, a segunda maior diversidade $H^{\prime}(0,76)$ e equitabilidade J' (0,63), além da segunda menor dominância k $(39,41 \%)$ (Tabela 3). Já nos horários de 06:00-12:00 e 12:00-18:00 horas a riqueza total (12 em ambos), diversidade H' $(0,56$ e 0,53 , respectivamente) e equitabilidade $J$ ' $(0,52$ e 0,49 , respectivamente) apresentaram valores inferiores, porém com dominância $\mathrm{k}$ acima de $68,57 \%$ (Tabela 3).

Das 26 espécies registradas para este trabalho, 16 delas mostraram-se como acidentais (registradas apenas uma vez) para pelo menos uma das áreas. A Tabela 4 demonstra que nas localidades Poço fundo e Jaguarizinho, respectivamente, onze e nove das espécies registradas, eram acidentais, com sete e oito espécies constantes e, duas e seis acessórias para estes locais. A espécie $P$. lineatus apresentou maior média de peso $(350,0 \mathrm{~g})$ e de comprimento $(45,0 \mathrm{~cm})$ enquanto $S$. brevipina apresentou menor média de peso (21,0 g) e G. gymnogenys menor média de comprimento $(10,15 \mathrm{~cm})$ (Tabela 4).

Os valores dos parâmetros físico-químicos da água demonstraram-se plenamente satisfatórios, não comprometendo a qualidade dos ambientes amostrados. A temperatura da água oscilou entre 10,00 e $25,50{ }^{\circ} \mathrm{C}$, o teor de oxigênio dissolvido variou entre 4,87 e 6,40 mg. $\mathrm{L}^{-1}$, o pH variou de 5,50 a 6,92 e a amônia total sempre ficou abaixo de $0,08 \mathrm{mg} / \mathrm{L} \mathrm{NH}_{3}$. A alcalinidade oscilou entre 10 e $27,25 \mathrm{mg} / \mathrm{L} \mathrm{CaCO}_{3}$ e a dureza entre 20 e $45 \mathrm{mg} . \mathrm{L}^{-1} \mathrm{CaCO}_{3}$ (Tabela 5).

Tabela 2. Diversidade de Shannon (H'); Equitabilidade de Pielou (J'); Riqueza Total (S); Número de Indivíduos (N) Dominância (k) para as diferentes épocas de coleta. Outubro $(\mathrm{O})$; dezembro (D); abril (Ab); agosto $(\mathrm{Ag})$.

Table 2. Shannon diversity (H'); Pielou evenness (J'): Total richness (S); Number of individuals $(\mathrm{N})$ and Dominance $(\mathrm{k})$ to the different times of collect. October (o); December (D); April (AP); August (Ag).

\begin{tabular}{ccccc}
\hline Valores & \multicolumn{4}{c}{ Estações do ano } \\
\cline { 2 - 5 } & O & D & Ab & Ag \\
\hline H' & 0,70 & 0,86 & 0,74 & 0,67 \\
J' & 0,60 & 0,67 & 0,61 & 0,60 \\
S & 15 & 19 & 16 & 13 \\
N & 156 & 146 & 135 & 135 \\
k (\%) & 55,13 & 50,00 & 45,18 & 52,59 \\
\hline
\end{tabular}

Tabela 3. Diversidade de Shannon (H'); Equitabilidade de Pielou (J'); Riqueza Total (S); Número de Indivíduos (N) e Dominância (k) para os diferentes horários de coleta.

Table 3. Shannon diversity (H'); Pielou evenness (J'): Total richness (S); Number of individuals $(\mathrm{N})$ and Dominance $(\mathrm{k})$ to the different hours of collect.

\begin{tabular}{ccccc}
\hline Valores & \multicolumn{4}{c}{ Horários de coleta (horas) } \\
\cline { 2 - 5 } & $\mathbf{0 : 0 0 - 6 : 0 0}$ & $\mathbf{6 : 0 0 - 1 2 : 0 0}$ & $\mathbf{1 2 : 0 0 - 1 8 : 0 0}$ & $\mathbf{1 8 : 0 0 - 2 4 : 0 0}$ \\
\hline H' & 1,07 & 0,56 & 0,53 & 0,76 \\
J' & 0,81 & 0,52 & 0,49 & 0,63 \\
S & 21 & 12 & 12 & 16 \\
N & 133 & 94 & 175 & 170 \\
k (\%) & 29,32 & 69,15 & 68,57 & 39,41 \\
\hline
\end{tabular}


Tabela 4. Constância da captura nos diferentes pontos de coleta e médias de peso e comprimento. Constante (A); acessória (B); acidental (C). Table 4. Capture Constance in the different points of collect and weight and length means.

\begin{tabular}{|c|c|c|c|c|}
\hline Espécie & Poço fundo & Jaguarizinho & Peso (g) & Comprimento $(\mathrm{cm})$ \\
\hline \multicolumn{5}{|l|}{ CHARACIFORMES } \\
\hline \multicolumn{5}{|l|}{ PARADONTIDAE } \\
\hline Apareiodon affinis & A & $\mathrm{A}$ & 21,68 & 13,01 \\
\hline \multicolumn{5}{|l|}{ CURIMATIDAE } \\
\hline Steindachenerina brevipina & A & A & 21,00 & 11,59 \\
\hline Steindachenerina biornata & $\mathrm{C}$ & - & 69,36 & 17,2 \\
\hline \multicolumn{5}{|l|}{ PROCHILODONTIDAE } \\
\hline Prochilodus lineatus & - & $\mathrm{B}$ & 350,00 & 45,00 \\
\hline \multicolumn{5}{|l|}{ ANOSTOMIDAE } \\
\hline Schizodon nasutus & $\mathrm{C}$ & $\mathrm{C}$ & 155,93 & 24,50 \\
\hline Leporinus striatus & - & $\mathrm{B}$ & 155,93 & 24,50 \\
\hline \multicolumn{5}{|l|}{ CHARACIDAE } \\
\hline \multicolumn{5}{|l|}{ Characinae } \\
\hline Astyanax jacuhiensis & A & A & 24,58 & 11,66 \\
\hline Astyanax fasciatus & - & $\mathrm{C}$ & - & - \\
\hline Galeocharax humeralis & $\mathrm{C}$ & - & - & - \\
\hline \multicolumn{5}{|l|}{ Serrasalminae } \\
\hline Serrasalmus maculatus & $\mathrm{C}$ & $\mathrm{C}$ & 279,30 & 23,40 \\
\hline \multicolumn{5}{|l|}{ ACESTRORHYNCHIDEAE } \\
\hline Acestrorhynchus pantaneiro & A & $\mathrm{B}$ & 134,29 & 24,13 \\
\hline \multicolumn{5}{|l|}{ ERYTHRINIDAE } \\
\hline Hoplias malabaricus & $\mathrm{C}$ & A & 258,56 & 27,50 \\
\hline Hoplias lacerdae & - & $\mathrm{C}$ & - & - \\
\hline \multicolumn{5}{|l|}{ SILURIFORMES } \\
\hline \multicolumn{5}{|l|}{ LORICARIIDAE } \\
\hline Hypostomus commersoni & A & A & 228,11 & 24,95 \\
\hline Hemiancistrus fuliginosos & A & A & 58,27 & 15,85 \\
\hline Hypostomus roseopuntactus & - & $\mathrm{C}$ & 177,15 & 26,90 \\
\hline Loricariichthys anus & - & $\mathrm{C}$ & - & - \\
\hline \multicolumn{5}{|l|}{ HEPTAPTERIDAE } \\
\hline Rhamdia quelen & B & B & 129,17 & 23,5 \\
\hline \multicolumn{5}{|l|}{ PIMELODIDAE } \\
\hline Pimelodus maculatus & A & $\mathrm{C}$ & 109,17 & 22,60 \\
\hline Iheringichthys labrosus & $\mathrm{C}$ & A & 83,82 & 22,40 \\
\hline Pimelodus atrobrunneus & $\mathrm{C}$ & B & 17,40 & 14,28 \\
\hline \multicolumn{5}{|l|}{ AUCHENIPTERIDAE } \\
\hline Auchenipterus osteomystax & $\mathrm{C}$ & - & - & - \\
\hline \multicolumn{5}{|l|}{ PERCIFORMES } \\
\hline \multicolumn{5}{|l|}{ SCIAENIDAE } \\
\hline Pachyurus bonariensis & $\mathrm{C}$ & $\mathrm{C}$ & - & - \\
\hline \multicolumn{5}{|l|}{ CICHLIDAE } \\
\hline Gymnogeophagus gymnogenys & $\mathrm{C}$ & B & 25,60 & 10,15 \\
\hline Crenicichla missioneira & $\mathrm{B}$ & A & 22,45 & 14,10 \\
\hline Crenicichla celidochilus & C & $\mathrm{C}$ & - & - \\
\hline
\end{tabular}


Copatti, C.E. et al.

Tabela 5. Valores médios dos parâmetros físico-químicos analisados na microbacia do rio Jaguari. Estação do ano: outubro (O), dezembro (D), abril (Ab), agosto (Ag).

Table 5. Means values of the parameters physical-chemical analyzed in the Microbasin of the River Jaguari. Time of year: October (o); December (D); April (AP); August (Ag).

\begin{tabular}{|c|c|c|c|c|c|c|c|c|}
\hline \multirow[t]{2}{*}{ Parâmetros } & \multicolumn{3}{|c|}{ Poço Fundo } & \multicolumn{5}{|c|}{ Jaguarizinho } \\
\hline & $\mathbf{O}$ & D & $\mathbf{A b}$ & Ag & $\mathbf{O}$ & D & $\mathbf{A b}$ & Ag \\
\hline Amônia total (mg.L. $\left.\mathrm{L}^{-1} \mathrm{NH}_{3}\right)$ & 0,01 & 0,01 & 0,01 & 0,08 & 0,01 & 0,01 & 0,01 & 0,08 \\
\hline $\mathrm{pH}$ & 6,00 & 5,50 & 6,00 & 6,50 & 6,45 & 6,92 & 6,00 & 6,50 \\
\hline Alcalinidade (mg.L $\mathrm{L}^{-1} \mathrm{CaCO}_{3}$ ) & 10,00 & 13,00 & 10,00 & 27,25 & 10,00 & 13,00 & 10,00 & 27,25 \\
\hline Dureza $\left(\mathrm{mg} . \mathrm{L}^{-1} \mathrm{CaCO}_{3}\right)$ & 20,00 & 32,00 & 30,00 & 45,00 & 20,00 & 32,00 & 30,00 & 45,00 \\
\hline Temperatura $\left({ }^{\circ} \mathrm{C}\right)$ & 16,00 & 23,00 & 10,00 & 11,80 & 18,00 & 25,5 & 10,00 & 14,00 \\
\hline Oxigênio Dissolvido (mg. $\left.\mathrm{L}^{-1}\right)$ & 6,25 & 6,40 & 5,25 & 4,87 & 5,20 & 6,25 & 4,87 & 4,87 \\
\hline
\end{tabular}

\section{Discussão}

Uma variação na riqueza de espécies e até mesmo no número de espécies pode ser esperada quando se considera a sazonalidade. Nesse trabalho, foram coletadas 19 espécies no mês de dezembro, enquanto que em agosto houve uma redução para 13 espécies. Esta variação é explicada por Lowe-McConnell (1975) que informa que em rios de regiões subtropicais, as comunidades são afetadas por mudanças estacionais que implicam na alteração dos parâmetros abióticos os quais interferem diretamente na dinâmica populacional das espécies.

Alguns estudos já foram efetuados na bacia do rio Uruguai, sendo que Weis et al. (1983) no levantamento da fauna ictiológica do rio Ibicuí-Mirim (RS), constataram a ocorrência de 81 espécies, Bossemeyer et al. (1985) coletaram um total de 1.345 peixes pertencentes a 53 espécies no rio Santa Maria (RS). Bertoletti et al. (1989a) estudando a ictiofauna do rio Uruguai em seu trecho superior entre os municípios de Aratiba e Esmeralda-RS, acusaram a ocorrência de 74 espécies, sendo que oito delas são consideradas restritas à região. Em trabalho desenvolvido no mesmo ano sobre a ictiofauna do rio Canoas, Bertoletti et al. (1989b) verificaram a existência de 53 espécies. Bertoletti et al. (1990) capturaram 5.638 exemplares de peixes pertencentes a 71 espécies na área de influência da usina hidrelétrica de Garabi, RS (rio Uruguai). Malabarba \& Isaia (1992), registraram 73 espécies para o sistema do rio Tramandaí, sendo que destas, 33 são citadas pela primeira vez para a referida área. Mais recentemente, Behr (2005) registrou a ocorrência de 111 espécies distribuídas em 29 famílias. No presente estudo foram encontradas 26 espécies contabilizando os dois trechos amostrados, mas devido à alta diversidade da bacia do rio Uruguai, este número tende a ser maior.

Ao contrário de rios mais caudalosos onde são registrados grande número de espécies, este estudo registrou menor riqueza de espécies, o que está de acordo com as dimensões dos ambientes de estudo (30-50 m de largura). Da mesma forma, outros autores em trabalhos realizados em ambientes de dimensões semelhantes, também registram menor riqueza. Azevedo et al. (2003) estudando o rio Felizardo, no município de Uruguaiana-RS, registraram 27 espécies, distribuídas em 11 famílias, através do método de pesca elétrica. Em análise da diversidade de espécies de peixes da área litorânea do trecho de transição entre o Ribeirão dos Veados e a represa de Jurumirim (alto rio Paranapanema, SP), foram registradas 26 espécies de peixes distribuídos em nove famílias, sendo a maioria de pequeno porte e pertencente à família Characidae. Em menor abundância, ocorreram representantes das ordens Siluriformes, Gymnotiformes, Perciformes e Synbranchiformes (Castro et al. 2003). No presente estudo, apesar de Loricariidae ter sido a família mais representativa com quatro espécies, as diferenças para outras famílias não evidentes.
Mansilha (1998) determinou para o rio Salso de Baixo, 26 espécies distribuídas em 10 famílias. Casatti et al. (2001), num estudo de peixes de rio do Parque Estadual Morro do Diabo, bacia do alto rio Paraná-SP, identificaram 22 espécies, pertencentes a 11 famílias. Barbieri et al. (2004), em pesquisa da composição íctica da represa de Ponte Nova em São Paulo, pertencente à bacia do alto rio Tietê, capturaram 1.507 exemplares distribuídos em 11 famílias e 24 espécies. Nos trabalhos realizados por Dabés et al. (2001) na Barragem do rio Juramento (Juramento, MG) foram capturados 1.105 peixes pertencentes a 15 espécies, distribuídas em duas ordens e sete famílias. Barrella \& Petrere (2003), em seus estudos sobre a ictiofauna da represa de Ponte Nova em São Paulo, entre 1994 e 1995, constataram a ocorrência de 14 espécies, sendo mais abundantes as espécies dos gêneros Astyanax, Cyphocharax e Geophagus. Através do conhecimento destes dados, ressalta-se a importância do curso d'água do rio Jaguari, o qual demonstra alta representatividade de espécies ícticas para a região.

A espécie mais abundante em nosso estudo, A. affinis, comumente conhecida na região como "canivete" apresenta, segundo Godoy (1975) ampla distribuição geográfica ocorrendo nas bacias do rio Paraná, Paraguai e Uruguai. A alimentação dessa espécie foi estudada por Sazima (1980) e Cassati et al. (2003), que a consideram algívora e por Hahn et al. (1997), que a descreveram como iliófaga. Santim et al. (2004), relatam que estudos de estágios iniciais de desenvolvimento são inexistentes, apesar de serem importantes para o entendimento do papel da espécie na cadeia alimentar. Em virtude de seu pequeno porte (até $15 \mathrm{~cm}$ ), não tem grande interesse comercial, mas é utilizada na alimentação por moradores ribeirinhos devido à abundância e fácil captura, sendo também é utilizada como isca para a pesca amadora.

Dentre os peixes neotropicais, os Loricariidae, comumente conhecidos como cascudos ocupam o segundo lugar em número de espécies, distribuem-se em toda a região neotropical e estão presentes em habitats muito variados, o que demonstra a sua capacidade adaptativa (Artoni \& Bertollo 1999). Segundo Britski (1972), o gênero Hypostomus, pertencente à família Loricariidae é o gênero de cascudos dominante nos rios brasileiros. H. commersoni foi a terceira espécie mais representativa deste estudo, sendo superada apenas por H. fuliginosos (Loricariidae) e A. affinis.

A família Curimatidae compreende diversas espécies popularmente conhecidas como birus, extremamente abundantes e de importância ecológica nas comunidades de peixes neotropicais, por serem animais de hábito detritívoro. São peixes de pequeno porte, não apresentam dentes e vivem agrupados próximo ao fundo, em águas abertas (Fink \& Fink 1978). Neste estudo, S. brevipina foi a quarta espécie mais abundante. 
Muitas espécies neotropicais consomem detrito: algumas são consumidoras ocasionais, enquanto outras, tais como as representantes das famílias Curimatidae (S. brevipina e S. biornata) e Loricaridae (H. commersoni e $H$. fuliginosos), são altamente especializadas (Bowen 1984). Nesse trabalho, relata-se que as quatro espécies mais abundantes compartilham o hábito detritívoro. Neste caso, a riqueza de espécies pode estar associada à estrutura do habitat (elevada quantidade de macrófitas aquáticas), que oferece condições adequadas para os detritívoros.

Além das dimensões, outros fatores influenciam na presença de espécies. A temperatura ideal é um desses fatores, sendo que da mesma forma que o melhor desenvolvimento para produção da maioria das espécies situa-se entre 25 e $28{ }^{\circ} \mathrm{C}$ (Schimittou 1993), a atividade dos peixes também pode ser associada à temperatura e à sazonalidade, uma vez que os dados encontrados pelo presente trabalho indicam uma menor riqueza e diversidade durante as estações mais frias do ano. Porém, isso nem sempre acontece, como demonstrado para outros rios pertencentes a bacia do rio Uruguai, como no rio Felizardo por Azevedo et al. (2003) que descreveram que o maior número de exemplares capturados ocorreu no outono com 1.530 indivíduos distribuídos em 23 espécies e 11 famílias, enquanto outros dados dão suporte aos resultados encontrados nesse trabalho, como descrito para a nascente do rio Imbaá, onde foi determinada a maior captura no verão, 272 indivíduos, distribuídos em 15 espécies e em 9 famílias (Saucedo et al. 2001) ou no rio Quaraí-Chico, onde a maior captura ocorreu na primavera, com 1.156 indivíduos, distribuídos em 23 espécies e 11 famílias (Correa 1998). Em relação às espécies encontradas para este trabalho, Saucedo et al. (2001), Azevedo et al. (2003) e Correa (1998) encontraram, respectivamente, cinco, sete e oito espécies também verificadas para este trabalho.

Lagler et al. (1962) e Lowe-McConnell (1975) mencionam que o período do dia é outro fator determinante da taxa de alimentação das espécies, havendo aquelas que se alimentam preferencialmente durante o período diurno, enquanto outras apresentam hábitos alimentares noturnos. Como foi demonstrado neste trabalho, ocorre uma incidência maior de espécies no período noturno, uma vez que as mesmas preferem desempenhar suas atividades na ausência de luminosidade.

As redes de espera refletem o tamanho de seus exemplares, ou seja, notou-se uma maior abundância de indivíduos pequenos em face de exemplares maiores nas coletas realizadas para este estudo. Casatti et al. (2001) contabilizam que aproximadamente 73,0\% das espécies e $96,0 \%$ dos indivíduos estudados em trabalho realizado em uma bacia do alto rio Paraná são de pequeno porte, reforçando a opinião de Casatti \& Castro (1998), que considera a predominância de peixes de pequeno porte como o único padrão geral com valor diagnóstico para a ictiofauna de rios sul-americanos. Ainda segundo os mesmos autores, associados ao pequeno porte destes peixes, seu grau relativamente elevado de endemismo e sua ocupação de microhabitat bastante específicos são fatores que acentuam ainda mais a necessidade de estabelecer estratégias para a conservação desses ambientes, o que é reforçado por Hook (1991), quando denota a importância de ecossistemas aquáticos, como os pequenos cursos d'água, por construírem áreas de criação, alimentação e refúgio de peixes.

Os índices de diversidades obtidos para o trecho do rio Jaguari são considerados baixos. Há sempre predomínio de poucas espécies. Essa diversidade baixa, embora com a riqueza de espécies elevada, pode refletir o grau de degradação ambiental a que está submetida atualmente a bacia hidrográfica. Segundo Odum (1988), a diversidade, muitas vezes, tende a ser reduzida em comunidades que sofrem estresse. Fatores como ausência de mata ripária, despejos de efluentes domésticos e agrotóxicos lixiviados de lavouras próximas, verificados no ambiente amostrado, tendem a acelerar tal estresse.
Peres-Neto et al. (1995) relatam que em rios tropicais, o aumento da diversidade ao longo do rio tem sido associado a um aumento na heterogeneidade de habitats, o qual, por sua vez, é relacionado principalmente a um aumento na vazão e na disponibilidade de abrigos e sugerem para peixes de rios tropicais um modelo de uso do microhabitat o qual prediz que a diversidade da comunidade depende da heterogeneidade de habitats. Para Ricklefs (2003) numa base regional, o número de espécies varia de acordo com a adequação das condições físicas, com a heterogeneidade de habitat, com o isolamento dos centros de dispersão e com a produtividade primária. Alterações dos hábitats, tais como, canalização das margens, destruição da vegetação ripária ou barramentos, além de introduções de espécies não-nativas e a deterioração da qualidade da água, exercem uma profunda e negativa influência no ecossistema, podendo causar variações na distribuição espaço-temporal na comunidade de peixes (Waite \& Carpenter 2000). Vieira \& Shibatta (2007), utilizaram os índices de diversidade para avaliar a qualidade ambiental do rio Esperança (Londrina, PR) e, assim como nesse trabalho, verificaram que uma alta dominância por poucas espécies reduz a diversidade e que a alta dominância foi relacionada com um grau mais elevado de poluição do rio.

Das 26 espécies coletas, 16 delas demonstraram-se acidentais para pelo menos umas das áreas, o que indica a ocorrência de uma espécie pode estar restrita (ou pelo menos fortemente relacionada) ao período de coleta e local de captura. De acordo com Lowe-McConnell (1975), com base em resultados de constância de ocorrência podemse caracterizar como duas ictiofaunas distintas, uma permanente (residente) e outra visitante (imigrante) sendo que uma comunidade residente pode ser aumentada por peixes imigrantes que se juntam aos residentes por algum tempo para se alimentar, reproduzir ou apenas passar pela comunidade. Garutti (1988) estudando a ictiofauna do córrego Barra Funda na bacia do Paraná, observou que 74,0\% das espécies registradas crescem e se reproduzem neste ambiente, indicando que o mesmo é propício ao desenvolvimento ontogenético; o restante das espécies foi considerado visitante.

A área de estudo sofre diversos impactos antrópicos, especialmente pela intensa atividade agrícola e pecuária. A falta de informações sobre a composição da ictiocenose no passado impossibilita sua comparação com a situação atual, considerando que o conhecimento da ictiofauna constitui uma etapa fundamental para a adoção de medidas de recuperação e manejo de ambientes já alterados.

\section{Agradecimentos}

Os autores agradecem ao Prof. Dr. Everton Rodolfo Behr da UFSM pelo auxílio na identificação dos exemplares

\section{Referências Bibliográficas}

ADAD, J.M.T. 1982. Controle químico de qualidade. Guanabara, Rio de Janeiro, $203 \mathrm{p}$.

AMORIN, M.C., PESSOA, M.A.R. \& MEDEIROS, K.L.S. 1983. Otimização da rede de monitoramento no rio Paraíba do Sul. Cadernos FEEMA, Série Congressos, 16(1):1-36.

ARTONI, R.F. \& BERTOLLO, L.A. 1999. Nature and distribution of constitutive heterochromatin in fishes, genus Hypostomus (Loricariidae). Genética, 106(3):209-214.

AZEVEDO, C.L.O., PESSANO, E.F.C., TOMASSONI, D.S., QUERO, M.V.M. \& QUEROL, E.C. 2003. Diversidade específica, densidade e biomassa da ictiofauna da nascente do arroio Felizardo, bacia do rio Uruguai Médio, Uruguaiana, RS, Brasil. Biodiversidade Pampeana, 1(1):35-45.

BARBIERI, G., JUNIOR, V.H., CAMARA, J.J.D., CAMPOS, E.C. \& GIAMAS, M.T.D. 2004. A ictiofauna da represa de ponte nova, Salesópolis (São Paulo) - bacia do alto Tietê. B. Inst. Pesca, 30(1):25-34. 
BARRELLA, W. \& PETRERE Jr., M. 2003. Fish community alterations due to pollution and damming in Tietê and Paranapanema rivers. River res. appl. 19(1):59-76.

BEHR, E.R. 2005. Estrutura da comunidade e alimentação da ictiofauna dominante do rio Ibicui, RS. Tese de Doutorado, Pontifícia Universidade Católica do Rio Grande do Sul - PUCRS, Porto Alegre.

BERTOLETTI, J.J. 1985. Aspectos sistemáticos e biológicos da ictiofauna do rio Uruguai. Véritas, 30(117):93-129.

BERTOLETTI, J.J., LUCENA, C.A.S., LUCENA, Z.M.S., MALABARBA, L.R. \& REIS, R.E. 1989a. Ictiofauna do rio Uruguai superior entre os municípios de Aratiba e Esmeralda, Rio Grande do Sul, Brasil. Comun. Mus. Cienc. PUCRS, Ser. zool. 32(48):3-42.

BERTOLETTI, J.J., LUCENA, C.A.S., LUCENA, Z.M.S., MALABARBA, L.R. \& REIS, R.E. 1989b. Ictiofauna do rio Canoas, sistema do rio Uruguai Superior, Campos Novos, Santa Catarina, Brasil. Comun. Mus. Cienc. PUCRS, Ser. zool. 32(49):43-75.

BERTOLETTI, J.J., LUCENA, C.A.S., LUCENA, Z.M.S., MALABARBA, L.R. \& REIS, R.E. 1990. Estrutura e composição da fauna de peixes na área de influência da futura Usina Hidrelétrica de Garabi, Rio Grande do Sul, Brasil. Comun. Mus. Cienc. PUCRS, Ser. zool. 3(2):33-97.

BOSSEMEYER, I.M.K., WEIS, M.L.C., BENEMANN, S.T. \& BIER, M.L.S. 1985. Ictiofauna do Rio Santa Maria, RS. Cienc. nat. 7:209-222.

BOWEN, S.H. 1984. Detritivory in neotropical fish communities. In Evolutionary ecology of Neotropical freshwater fish (T.M. Zaret, ed.). W. Junk, Dordrecht, p. 59-66.

BOYD, C.E. 1982. Water quality management for pond fish culture. Elsevier, Amsterdam, $318 \mathrm{p}$.

BRITSKI, H.A. 1972. Poluição e piscicultura. CIBPU; Faculdade de Saúde Pública da USP; Instituto de Pesca, São Paulo, p. 103.

BUCKUP, P.A., MENEZES, N.A. \& GHAZZI, M.S. 2007. Introdução. In Catálago das espécies de peixes de água doce do Brasil (P.A. Buckup, N.A. Menezes \& M.S. Ghazzi, eds.). Museu Nacional, Rio de Janeiro, p. 11-14.

CASATTI, L. \& CASTRO, R.M.C. 1998. A fish community of the São Francisco River headwaters riffles, southeastern Brazil. Ichthyol. Explor. Freshwaters, 9(3):229-242.

CASATTI, L., CASTRO, R.M.C. \& LANGEANI, F. 2001. Peixes de riacho do Parque Estadual Morro do Diabo, bacia do alto rio Paraná, SP. Biota Neotropica 1(1): http://www.biotaneotropica.org.br/v1n12/pt/ fullpaper?bn00201122001+pt (último acesso em 22/04/2009).

CASTRO, R.J., FORESTI, F. \& CARVALHO, E.D. 2003. Composição e abundância da ictiofauna na zona litorânea de um tributário, na zona de sua desembocadura no reservatório de Jurumirim, Estado de São Paulo, Brasil. Inst. Bio. 25(1):63-70.

CORREA, F.V. 1998. Determinação quantitativa das populações ícticas do arroio Quarai-Chico, em área de mata ciliar, bacia do rio Uruguai, Pampa Brasileiro. Uruguaiana. Monografia de Graduação, PUCRS, Uruguaiana.

DABÉS, M.B.G.S., SANTOS, G.B., RATTON, T.F. \& MEDEIROS, G.R. 2001. Estudo da Ictiofauna na Barragem do rio Juramento, Juramento, MG, Brasil. Unimontes cient. 1(1):75-85.

DAJOZ, R. 1973. Ecologia geral. EDUSP, São Paulo, 474 p.

Di PERSIA, D.H. \& NEIFF, J.J. 1980. The Uruguay river system. In The ecology of river systems (B.R. Davies \& K.F. Walker, eds.). Dr. Junk Publications, Netherlands, p. 599-621.

FINK, W.I. \& FINK, S. 1978. A Amazônia Central e seus peixes. Acta Amazon. 8(4):19-42.

GARUTTI, V. 1988. Distribuição longitudinal da ictiofauna em um córrego da região noroeste do estado de São Paulo, bacia do rio Paraná. Rev. bras. biol. 48(4):747-759.

GODOY, M.P. 1975. Peixes do Brasil - Subordem Characoidei: Bacia rio Mogi Guassu. Franciscana, Piracicaba.

HAHN, N.S., AGOSTINHO, A.A., GOMES, L.C. \& BINI, L.M. 1997. Estrutura trófica. In A planície de inundação do alto Rio Paraná: aspectos físicos, biológicos e socioeconômicos (A.E.A.M. Vazzoler, A.A. Agostinho \& N.S. Hahn, eds.). EDUEM, Nupélia; Maringá, p. 229-248.

HICKSON, R.G., MARANHÃO, T.C.F., VITAL, T.S. \& SEVERI, W. 1995. Método para a caracterização da ictiofauna em estudos ambientais. In Manual de avaliação de impactos ambientais (P.A. Juchem, ed.). SEMA/ IAP/GTZ, Curitiba, p. 1-8.

HOOK, J.H. 1991. Seasonal variation in the relative abundance and species diversity of fishes in South Bay. Contr. Mar. Sci. 32(1):127-141.

LAGLER, K.F., BARDACH, J.E. \& MILLER, R.R. 1962. Ichthyology. John Wiley \& Sons, Inc., New York, 454 p.

LOWE-McCONNELL, R.H. 1975. Fish communiteis in tropical freshwaters. Longman, New York, 337 p.

MALABARBA, L.R. \& ISAIA, E.A. 1992. The fresh water fish fauna of the rio Tramandaí drainage, Rio Grande do Sul, Brazil, with a discussion of its historical origin. Comun. Mus. Cienc. PUCRS, Ser. zool. 5(12):197-223.

MALABARBA, L.R. \& REIS, R.E. 1987. Manual de técnicas para a preparação de coleç̃oes zoológicas. Soc. bras. zool., 36(1):1-14.

MANSILHA, L.V.Q. 1998. Diversidade específica e estimativa da densidade e biomassa da ictiofauna do Arroio Salso de Baixo, na região de Uruguaiana, bacia do Rio Uruguai, RS, Brasil. Monografia de Conclusão do Curso, PUCRS, Uruguaiana.

ODUM, E.P. 1988. Ecologia. Guanabara, Rio de Janeiro, 434 p.

PERES-NETO, P.R., BIZERRIL, C.R.S.F. \& IGLESIAS, R. 1995. An overview of some aspects ofriver ecology: a case study on fish assemblages distribution in an eastern Brazilian coastal river. In Estrutura, funcionamento e manejo de ecossistemas brasileiros (F.A. Esteves, ed.). PPGE - UFRJ, Rio de Janeiro, p. 317-334.

RICKLEFS, R.E. 2003. A economia da natureza. Guanabara Koogan S.A, São Paulo, 505 p.

SANTIM, M., BIALETZKI, A. \& NAKATANI, K. 2004. Mudanças ontogênicas no trato digestório e dieta da Apareiodon affinis. Rev. Bras. Biol. 26(3):291-298.

SAUCEDO, L.S., QUEROL, E., QUEROL, M., MARTINS, S.S. \& BRANDLI, M.R.S. 2001. Dinâmica das populações ícticas do arroio Imbaá (nascente) bacia do rio Uruguai médio, Uruguaiana, RS, Brasil. In Livro dos resumos do I Salão de Iniciação Científica da PUCRS Campus de Uruguaiana. PUCRS, Uruguaiana, p. 9.

SAZIMA, I. 1980. Behavior of two Brazilian species of Paradontid fishes, Apareiodon piracicabae and Apareiodon ibitiensis. Copeia, 1:166-169.

SCHIMITTOU, H.R. 1993. Produção de peixes em alta densidade em tanquesrede de pequeno volume. Associação Americana de Soja; Mogiana Alimentos, Campinas, 78 p.

VIEIRA, A.L. 1984. Aspectos do metabolismo lipídico do curimbatá Prochilodus scrofa (Steindachner, 1881) no estádio de repouso gonadal. Bol. Inst. Pesca, 11(1):63-68.

VIEIRA, D.B. \& SCHIBATTA, O.A. 2007. Peixes como indicadores de qualidade ambiental do ribeirão Esperança, município de Londrina, Paraná, Brasil. Biota Neotropica 7(1): http://www.biotaneotropica.org.br/ v7n1/pt/fullpaper?bn01407012007+pt. (último acesso em 22/04/2009).

WAITE, I.R. \& CARPENTER, K.D. 2000. Associations among fish assemblage structure and environmental variables in Willamette basin streams, Oregon. Transactions of the American Fisheries Society, 129(5):754-770.

WEIS, M.L.C., BOSSEMEYER, I.M.K., BIER, M.L.S. \& LIPPOLD, H.O. 1983. Inventário da fauna ictiológica do rio Ibicui-Mirim, RS. Cienc. nat. 5:135-152. 